\title{
Editorial
}

Digestion

\section{The Need for Resistance Surveillance and Antimicrobial Susceptibility Testing of Helicobacter pylori}

\section{Erik-Oliver Glocker}

National Reference Centre for Helicobacter pylori, Department of Medical Microbiology and Hygiene, Institute of Medical Microbiology and Hygiene, University Medical Centre Freiburg, Freiburg, Germany

Infections with Helicobacter pylori may result in complications such as peptic ulcer disease, gastric adenocarcinoma or mucosa associated lymphoid tissue lymphoma. Thus, European guidelines recommend treatment of infected individuals by administration of empiric therapies, usually consisting of 2 antimicrobials in combination with a proton pump inhibitor [1]. Antimicrobial resistances to $H$. pylori, however, are increasing worldwide and hinder the success of such empirical treatments. The work by Narravo-Jarabo et al. [2], published in the last issue of Digestion, shows that in the Spanish region of Andalusia, primary resistances to clarithromycin and levofloxacin may vary considerably from individual to individual. Their multicenter study revealed that primary resistance to the key antibiotic - clarithromycin - was ranging from 11 to $25 \%$, primary resistance to levofloxacin was ranging from 7 to $17 \%$. The data also underscore the need for studies keeping the development of antimicrobial resistances under surveillance. Continuous surveillance studies that monitor the present state of antimicrobial resistance are indispensable to establish updated guidelines on first-line eradication treatments. Remarkably, Narravo-Jarabo et al. [2] tested the resistance to clarithromycin and levofloxacin at the molecular genetic level by identification of mutations conferring resistance.
Genotypic susceptibility testing is not only quicker and most likely more cost-effective, but also less vulnerable to contaminations and easier to standardize when compared with classical microbiological methods including culture and phenotypic testing. Molecular genetic testing may also enable resistance testing of larger cohorts as no live bacteria are required and no logistic constraints need to be considered.

The manuscript by Boltin et al. [3], also published in the last issue of Digestion, challenges the usefulness of repeated antimicrobial susceptibility testing in patients who had already undergone individualized susceptibility-guided eradication therapies. Their data show that repeated antimicrobial susceptibility testing may result in new resistances, particularly, in the case of $H$. pylori that were primarily susceptible to metronidazole, clarithromycin and levofloxacin. In $H$. pylori that were already resistant to two of these antimicrobials, the likelihood of developing further resistances was low. Such patients may not benefit from another endoscopy with taking gastric tissue samples for microbiological examination. Furthermore, eradication success was quite low given that the patients were administered susceptibility-guided therapies. This confirms that several other factors such as patients' compliance and individual genetic

\section{KARGER 125}

(c) 2015 S. Karger AG, Base

$0012-2823 / 15 / 0923-0173 \$ 39.50 / 0$ 
backgrounds (e.g. variations in genes encoding IL- $1 \beta$ or cytochrome $\mathrm{P} 450$ 2C19) may interfere with treatment success.

International guidelines recommend susceptibility testing after a second failure of empirical treatments [1]. Given the data presented in these 2 articles, susceptibility testing at earlier stages might be of more benefit to affected patients and may help physicians to select an appropriate eradication treatment. It needs to be discussed in future whether further susceptibility testing is useful once a resistance-guided treatment has already been unsuccessful.

\section{References}

1 Malfertheiner P, Megraud F, O'Morain CA, Atherton J, Axon AT, Bazzoli F, Gensini GF, Gisbert JP, Graham DY, Rokkas T, El-Omar EM, Kuipers EJ; European Helicobacter Study Group: Management of Helicobacter pylori infection - the Maastricht IV/Florence Consensus Report. Gut 2012;61:646-664.
2 Navarro-Jarabo JM, Fernández-Sánchez $F$, Fernández-Moreno N, Hervas-Molina AJ, Casado-Caballero F, Puente-Gutierrez JJ, Pallares-Manrique H, Rodríguez-Ramos C, Fernández-Gutierrez C, Pérez-Aisa A, RivasRuiz F, Montiel Quezel-Guerraz N: Prevalence of primary resistance of Helicobacter pylori to Clarithromycin and levofloxacin in Southern Spain. Digestion 2015;92:78-82.
3 Boltin D, Ben-Zvi H, Perets TT, GingoldBelfer R, Dickman R, Niv Y: Appropriateness of repeating Helicobacter pylori culture and susceptibility testing following failure of individualized antibiotic therapy. Digestion 2015;92:66-72. 